REVISTA CHILENA DE NEUROPSICOLOGIAA UNIVERSIDAD DELAFRONTERA

\section{Revista Chilena de Neuropsicología}

ISSN: 0718-0551

editor@neurociencia.cl

Universidad de La Frontera

Chile

Silva, Marisol; Solis, Silvia; Pérez, Elba; Martínez, Oscar A.

Influencia de las hormonas sexuales en mujeres con esclerosis múltiple durante la memoria de trabajo

y la activación cerebral medida con mapeo electroencefalográfico

Revista Chilena de Neuropsicología, vol. 7, núm. 2, 2012, pp. 85-90

Universidad de La Frontera

Temuco, Chile

Disponible en: http://www.redalyc.org/articulo.oa?id=179324185008

Cómo citar el artículo

- Número completo

- Más información del artículo

Página de la revista en redalyc.org

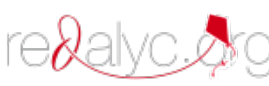

Sistema de Información Científica

Red de Revistas Científicas de América Latina, el Caribe, España y Portugal Proyecto académico sin fines de lucro, desarrollado bajo la iniciativa de acceso abierto 


\title{
Influencia de las hormonas sexuales en mujeres con esclerosis múltiple durante la memoria de trabajo y la activación cerebral medida con mapeo electroencefalográfico
}

\author{
Influence of sex hormones in women with MS during the working memory and brain activation \\ measured by electroencephalogram mapping
}

\author{
Marisol Silva ${ }^{1 *}$, Silvia Solis ${ }^{2}$, Elba Pérez $^{2}$, Oscar A. Martínez ${ }^{3}$ \\ 1 Escuela de Medicina, Universidad de Celaya. Guanajuato, México. \\ 2 Departamento de Ciencias Médicas de la División de Ciencias de la Salud, Campus León, Universidad de Guanajuato. Guanajuato, México. \\ 3 Universidad Politécnica del Estado de Guanajato. Guanajato, México.
}

\section{Resumen}

La Esclerosis Múltiple es una enfermedad inflamatoria desmielinizante del Sistema Nervioso Central, que se presenta con más frecuencia en mujeres, lo cual sugiere que las hormonas sexuales parecen modular la manifestación de la sintomatología. La actividad electroencefalografíca no ha sido evaluada en mujeres con Esclerosis Múltiple durante el procesamiento de Memoria de Trabajo y su estado hormonal. Los registros se realizaron en las fases folicular y lútea del ciclo menstrual de manera simultánea la tarea cognitiva y el electroencefalograma, además se correlacionaron con los niveles hormonales. La fase lútea mostró una ejecución mejor con un mayor número de categorías alcanzadas $(p<0.004)$, un menor número de errores perseverativos $(p<0.003)$ y un mayor número de ensayos para alcanzar una categoría $(p<0.011)$, se asoció con un aumento de la actividad de Theta $(p<0.018)$ y Alfa 2 ( $p<0.000)$. El 92\% de la variabilidad en el número de ensayos de la tarea, fue explicada por la actividad de Theta y Alfa 2 , Hormona Luteinizante y Estrona en la fase folicular, en la fase lútea el $90 \%$ de la variabilidad fue explicada por la actividad de Theta, Hormona Luteinizante, Progesterona y Estradiol. La Memoria de trabajo mejora en la fase lútea del ciclo menstrual, sugiriendo que la progesterona parece facilitar actividad de Theta.

Palabras clave: esclerosis múltiple, memoria de trabajo, hormonas sexuales, actividad cerebral

\begin{abstract}
Multiple Sclerosis is an inflammatory demyelinating CNS disease that occurs most often in women, suggesting that sex hormones appear to modulate the manifestation of symptoms. EEG activity has not been evaluated in women with MS during the processing of MT and hormonal status. The recordings were made in the follicular and luteal phases of the menstrual cycle a simultaneous cognitive task and EEG also were correlated with hormone levels. The luteal phase showed a better performance with a greater number of categories achieved $(p<0.004)$, fewer perseverative errors $(p<0.003)$ and greater number of trials to reach a category $(p$ $<0.011)$ was associated with increased theta activity $(p<0.018)$ and Alpha $2(p<0.000) .92 \%$ of the variability in the number of trials of the task was explained by the activity of Theta and Alpha 2, LH and estrone levels in the follicular phase, luteal phase, $90 \%$ of the variability was explained by the activity of theta, $\mathrm{LH}$, progesterone and estradiol. The MT improvement in the luteal phase of the menstrual cycle, suggesting that progesterone seems to facilitate Theta activity.
\end{abstract}

Keywords: Multiple Sclerosis, working memory, sex hormones, brain activity

\footnotetext{
* Correspondencia: msilva@udec.edu.mx. Escuela de Medicina de la Universidad de Celaya. Carretera Panamericana Km. 269. CP 37350. Celaya, Guanajuato, México. Teléfono: +52 (461) 6139099.

Recibido: 28-11-11. Revisión desde: 22-03-12. Aceptado: 05-05-12
}

DOI: 10.5839/rcnp.2012.0702.08 


\section{Introducción}

La Esclerosis Múltiple (EM) es una enfermedad inflamatoria desmielinizante del Sistema Nervioso Central (SNC) (Bruck, 2005).

En la EM se ha observado que las mujeres son más susceptibles que los hombres en una relación 2:1 y que existen diferencias sexuales en el curso de la enfermedad, (Beatty, 2002; Duquette \& Girad, 1993; Yonker et al., 2003) y lo cual sugiere una posible modulación por las hormonas sexuales, (Sandyk, 1996; Pozzilli et al., 1999; Tomassini et al., 2005; Holmqvist et al., 2006) ya que algunas evidencias clínicas sugieren que existe una influencia de estas sobre la actividad del sistema inmune en la enfermedad (Takao, Flink, Lee, \& Ying, 2004; Lang, 2004; Trenova, Manova, \& Zahariev, 2004). Del mismo modo, se ha observado que los niveles hormonales correspondientes a la fase folicular y lútea del ciclo menstrual son similares a los niveles hormonales que muestran las mujeres sanas (Pozzilli et al., 1999, Solís-Ortiz, Guevara, \& Corsi, 2004).

En el embarazo, las mujeres con EM muestran un efecto favorable sobre la enfermedad (Drew \& Chavis, 2000; Confavreux \& Hutchinson, 1998; Vukusic \& Confavreux, 2006; Achiron et al., 2004). Durante el ciclo menstrual, se ha reportado un deterioro en los síntomas de la enfermedad que preceden al inicio de la menstruación (Zorddrager \& De Keyser, 1997).

Los pacientes muestran un déficit en la memoria a corto plazo, conocida como memoria de trabajo (Esposito et al., 1996), una disfunción en este nivel lleva a una distorsión en la planeación y ejecución de tareas, así como a un comportamiento rígido y perseverativo que puede ser evaluado por Wisconsin Card Sorting Test (WCST), una prueba neuropsicologica ampliamente utilizada para evaluar alteraciones del lóbulo frontal y que contiene un fuerte componente de memoria de trabajo (Heaton, 1981; Everett, James, Lavoie, \& Karyme, 2001). Estudios en electroencefalografía (EEG) muestran que los pacientes con EM y alteración de la memoria, tuvieron un incremento de las bandas Delta y Theta en las regiones frontotemporales (Leocani \& Locatelli, 2000). Sin embargo, estos estudios no consideraron las diferencias debidas al género, ya que se sabe existen diferencias en las oscilaciones de la actividad EEG en mujeres con respecto a las etapas del ciclo menstrual.

\section{Método}

Se evaluaron pacientes con diagnóstico de EM según los criterios estandarizados de Poseer (1983). Se comparó con un grupo control de mujeres sanas

Se incluyó a pacientes del sexo femenino, de 20-45 años de edad, con ciclos menstruales regulares, no fumadoras, con un tiempo de diagnóstico de 5 a 10 años, escala de EDSS de 1-6 (Kurtzke, 1983) y que no ingirieran fármacos que actúan en el Sistema Nervioso Central.

Se entregó una carta de Consentimiento Informado acorde a los criterios de declaración de Helsinki.

\section{Determinación de los niveles hormonales}

Se les tomó $10 \mathrm{ml}$ de sangre venosa periférica, las muestras sanguíneas se obtuvieron 24 horas después de la administración del b-interferón. Se midieron los niveles hormonales de Estradiol, Estrógenos, Progesterona, Hormona Folículo Estimulante (FSH), y Hormona Luteinizante (LH).

Tarea de memoria de trabajo

Se utilizó la prueba "Wisconsin Card Sorting Test" (Heaton, 1981) para evaluar la memoria de trabajo durante el mapeo EEG en la fase folicular y lútea del ciclo menstrual, 24 horas después de la administración del beta interferón.

\section{Mapeo EEG}

Se generaron los mapas EEG para cada una de las bandas por medio del software Neuroscan LT. Cada mapa representa el porcentaje total de actividad que ocurre, cuenta con una escala a color que ubica la actividad en $V$ donde el color rojo es el punto más activo y el azul representa ausencia de actividad.

\section{Análisis estadístico}

Se realizó una prueba de $t$ de student para comparar cada banda del EEG, en la línea base y durante la ejecución de la tarea en las dos fases del ciclo, en los dos grupos; y un análisis de regresión, para estudiar los factores asociados a la actividad $E E G$, niveles hormonales y ejecución de la tarea.

\section{Resultados}

\section{Número de Categorías Alcanzadas}

Durante la ejecución de la tarea, el numero de las pacientes fue significativamente menor en la fase folicular ( $t=-3.067$; $p<0.006)$ y lútea $(t=-3.630 ; p<.001)$ del ciclo menstrual (ver figura 1).

Tabla 1. Características demográficas y clínicas de las pacientes.

\begin{tabular}{|c|c|c|c|c|c|}
\hline \multirow{3}{*}{ Características } & \multicolumn{4}{|c|}{ Mujeres } & \multirow{3}{*}{$p$} \\
\hline & \multicolumn{2}{|c|}{$\begin{array}{c}\text { Pacientes } \\
(n=13)\end{array}$} & \multicolumn{2}{|c|}{$\begin{array}{l}\text { Mujeres sanas } \\
\qquad(n=13)\end{array}$} & \\
\hline & $x$ & DS & $x$ & DS & \\
\hline Edad (años) & 33.86 & 5.82 & 34.51 & 6.14 & 0.190 \\
\hline IMC (kg/m2) & 25.60 & 1.10 & 24.10 & 0.93 & 0.180 \\
\hline Número de Embarazos & 1.30 & 0.39 & 0.70 & 0.026 & 0.097 \\
\hline Escolaridad (años) & 11.08 & 3.5 & 12.03 & 3.5 & 0.440 \\
\hline $\begin{array}{l}\text { Puntos Barthel } \\
\text { (incapacidad) }\end{array}$ & 83.56 & 15.41 & 100 & 0.50 & 0.0009 \\
\hline $\begin{array}{l}\text { Evolución de la } \\
\text { enfermedad (años) }\end{array}$ & 7.24 & 1.85 & & & \\
\hline $\begin{array}{l}\text { Puntos EDSS } \\
\text { (discapacidad) }\end{array}$ & 4.27 & 1.27 & & & \\
\hline
\end{tabular}


Tabla 2. Ejecución de la tarea de memoria de trabajo de las Mujeres Sanas.

\begin{tabular}{|c|c|c|c|c|c|}
\hline \multirow{3}{*}{ Variables } & \multicolumn{5}{|c|}{ Fases del Ciclo Menstrual de las Mujeres Sanas } \\
\hline & \multicolumn{2}{|c|}{ Folicular } & \multicolumn{2}{|c|}{ Lútea } & \multirow{2}{*}{$p$} \\
\hline & $x$ & DS & $x$ & DS & \\
\hline $\begin{array}{l}\text { Número de Categorías } \\
\text { Alcanzadas }\end{array}$ & 5.12 & 1.67 & 5.83 & 0.38 & ns \\
\hline Número de Errores & 8.75 & 4.86 & 3.16 & 0.03 & 0.001 \\
\hline \multicolumn{6}{|l|}{ Perseverativos } \\
\hline $\begin{array}{l}\text { Número de Errores para } \\
\text { mantener un Criterio }\end{array}$ & 29.33 & 19.20 & 23.75 & 14.11 & ns \\
\hline $\begin{array}{l}\text { Número de ensayos para } \\
\text { completar cada Categoría }\end{array}$ & 15.83 & 4.74 & 10.33 & 2.84 & 0.001 \\
\hline Nota. Los datos son expre & omo $n$ & ia \pm de & está & r. ${ }^{*} p<$ & \\
\hline
\end{tabular}

\begin{tabular}{|c|c|c|c|c|c|}
\hline \multirow{3}{*}{ Variables } & \multicolumn{5}{|c|}{$\begin{array}{l}\text { Fases del Ciclo Menstrual de las Mujeres con } \\
\text { EM }\end{array}$} \\
\hline & \multicolumn{2}{|c|}{ Folicular } & \multicolumn{2}{|c|}{ Lútea } & \multirow{2}{*}{$p$} \\
\hline & $x$ & DS & $x$ & DS & \\
\hline $\begin{array}{l}\text { Número de Categorías } \\
\text { Alcanzadas }\end{array}$ & 2.40 & 1.65 & 4.02 & 1.70 & 0.004 \\
\hline $\begin{array}{l}\text { Número de Errores Perseve- } \\
\text { rativos }\end{array}$ & 23.16 & 14.23 & 11.25 & 7.42 & 0.003 \\
\hline $\begin{array}{l}\text { Número de Errores para } \\
\text { mantener un Criterio }\end{array}$ & 45.91 & 21.52 & 42.08 & 19.39 & 0.423 \\
\hline $\begin{array}{l}\text { Número de ensayos para } \\
\text { completar cada Categoría }\end{array}$ & 76.50 & 26.87 & 55.33 & 33.34 & 0.011 \\
\hline
\end{tabular}

Número de Errores Perseverativos

Obtenido por las mujeres con EM fue significativamente mayor en la fase folicular $(t=3.321 ; p<0.003)$ y en la fase lútea $(t=3.637 ; p<0.001)$ del ciclo menstrual (ver figura 2$)$.

\section{Número de Errores para mantener un Criterio}

No mostró diferencias significativas en la fase folicular mientras que en la fase lútea se observo un número significativamente mayor ( $t=2.647 ; p<0.01$ ) (ver figura 3).

\section{Número de Ensayos para Completar una Categoría}

Las mujeres con EM obtuvieron un número significativamente mayor de ensayos para completar una categoría en la fase folicular $(t=7.637 ; p<0.000)$ y en la fase lútea $(t=4.658$; $p<0.000)$ del ciclo menstrual comparada con la mismas fases del ciclo de las mujeres sanas (ver figura 4).

La comparación de la ejecución de la tarea entre las mujeres con EM y las mujeres sanas mostró siempre un desempeño deficiente (ver tabla 2).

Ejecución de la tarea de MT entre las fases folicular y lútea de las pacientes con EM

Se observó una ejecución significativamente mejor durante la fase lútea del ciclo, con un mayor número de categorías alcanzadas $(t=3.39 ; p<0.004)$, un menor número de errores perseve- rativos $(t=3.85 ; p<0.003)$ y con un menor número de ensayos para completar una categoría $(t=3.045 ; p<0.011)$ (ver tabla 3 ).

La actividad EEG de las pacientes con EM durante la ejecución de la tarea en la fase folicular mostró una activación significativamente menor de las ondas lentas Delta $(p<0.002)$, Theta $(p<0.004)$ y una activación mayor de la onda rápida Beta 2 ( $p<0.000)$ comparada con la actividad EEG de las mujeres sanas. Durante la fase lútea la actividad de las bandas EEG mostró un aumento significativo en la banda Theta $(p<0.028)$ mientras que la banda Beta 2 disminuyo su actividad $(p<0.001)$. Las bandas EEG Alfa 1 , Alfa 2 y Beta 1 no mostraron diferencias significativas en ninguna de las dos fases (ver figura $5)$.

Comparación de las fases folicular y lútea del ciclo menstrual entre las pacientes con EM

Cuando se comparó la actividad EEG durante la ejecución de la tarea en las fases folicular y lútea del ciclo menstrual de las pacientes con EM, la fase lútea mostró una actividad mayor de la banda Theta $(p<0.018)$ y Alfa $2(p<0.000)$ mientras que la banda Beta 2 tuvo una actividad significativamente menor $(p<0.001)$ comparada con la fase folicular del mismo grupo de pacientes (ver figura 6).

\section{Mapeo espectral de la Actividad EEG en las mujeres con EM}

El mapeo espectral durante la ejecución de la tarea de memoria de trabajo en las mujeres con EM mostró en la fase lútea del ciclo una mayor actividad de la banda Theta y Alfa 2, mientras Beta 2 mostró una menor actividad (ver figura 7).

El mapeo espectral durante la ejecución de la tarea de memoria de trabajo en las mujeres sanas mostró un aumento de la actividad EEG de las bandas Delta, Theta, Alfa y Beta en fase lútea del ciclo menstrual (ver figura 8).

\section{Número de Ensayos de la Tarea como Variable Dependiente}

\section{Fase Folicular}

Los resultados de este análisis mostraron que el $92 \%$ de la variabilidad en el número de ensayos necesitados para alcanzar el éxito $\left(R^{2}=.966, p<0.001\right)$ fue explicada por la actividad de las bandas de theta y alfa 2 combinadas con los niveles hormonales de LH y estrona durante la fase folicular. El número de ensayos se asoció positivamente con la banda de alfa 2 $(p<0.000)$ y con estrona $(p<0.001)$, y negativamente con la actividad de theta $(p<0.001)$ y con LH $(p<0.003)$.

\section{Fase Lútea}

El $90 \%$ de la variabilidad en el número de ensayos necesitados para alcanzar el éxito $\left(R^{2}=.953, p<0.002\right)$ durante la fase lútea, también fue explicada por la actividad de la banda de theta combinada con los niveles hormonales de progesterona, estradiol, LH. El número de ensayos durante esta etapa se asoció positivamente con progesterona $(p<0.011)$ y LH $(p<0.000)$, mientras que la actividad theta y los niveles de estradiol se asociaron negativamente $(p<0.000),(p<0.015)$. 
Figura 1. Memoria de trabajo y número de categorías alcanzadas.

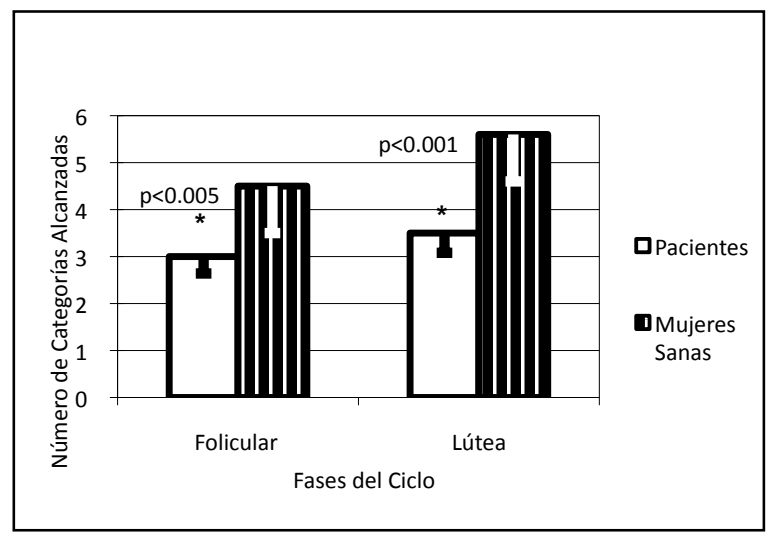

Nota. Las barras muestran la media \pm error estándar del Número de Categorías Alcanzadas en las fases folicular y lútea del ciclo menstrual en ambos grupos de mujeres.

Figura 3. Memoria de trabajo y errores para mantener un criterio.

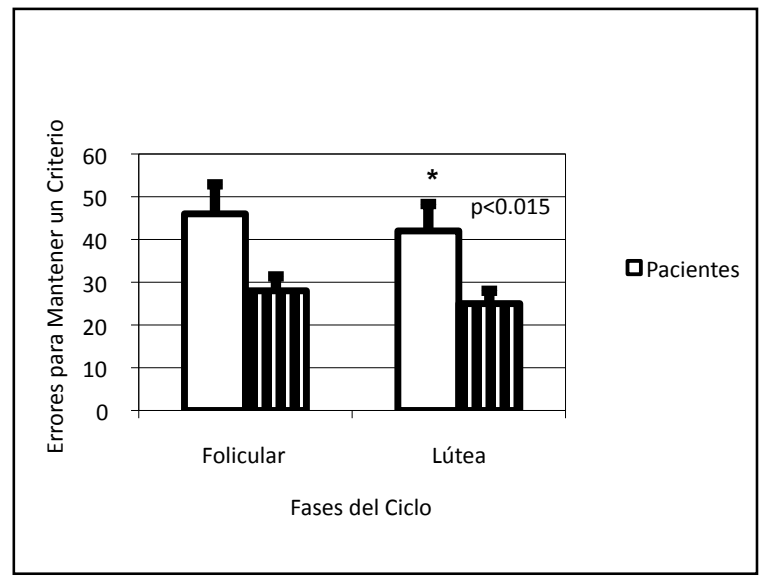

Nota. Las barras muestran la media \pm estándar del Número de Errores para Mantener un Criterio en las fases folicular y lútea del ciclo menstrual en ambos grupos.

Figura 5. Actividad cerebral durante la ejecución de la memoria de trabajo.
Figura 2. Memoria de trabajo y errores perseverativos en ambos grupos.

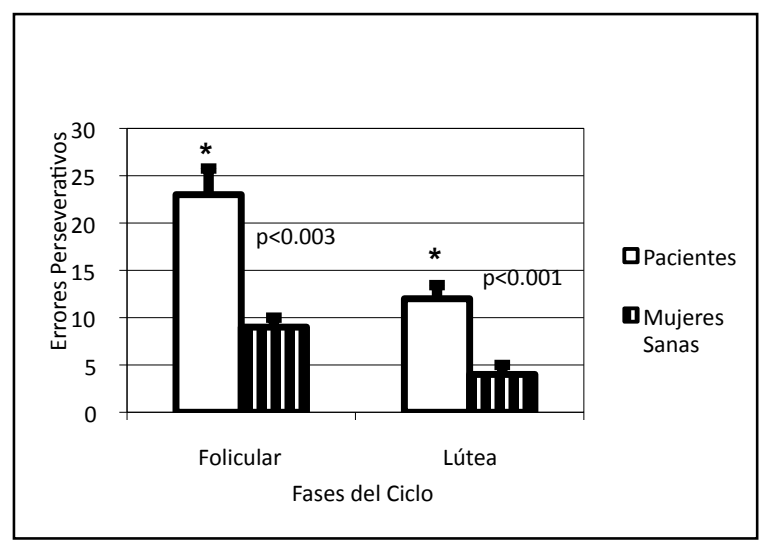

Nota. Las barras muestran la media \pm estándar del Número de Errores Perseverativos en las fases folicular y lútea del ciclo menstrual en ambos grupos de mujeres.

Figura 4. Memoria de trabajo y número de ensayos para completar una categoría.

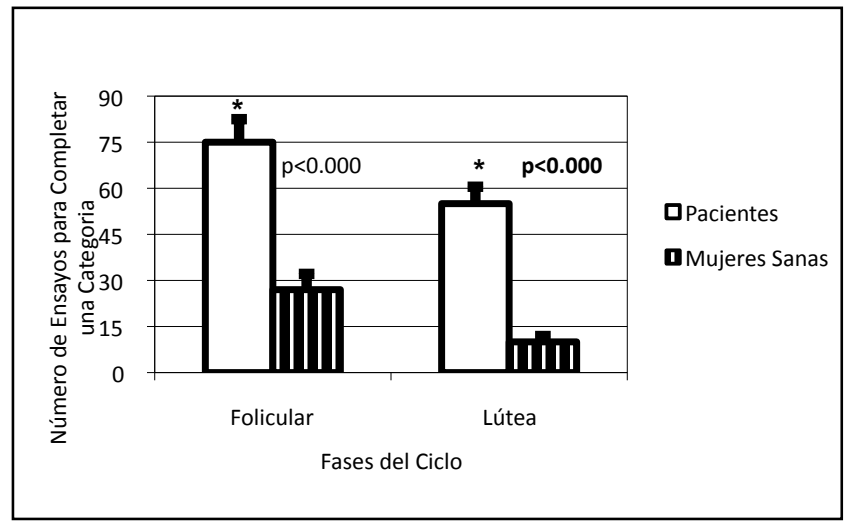

Nota. Las barras muestran la media \pm estándar del Número de Ensayos para Alcanzar una Categoría, en ambos grupos de mujeres.

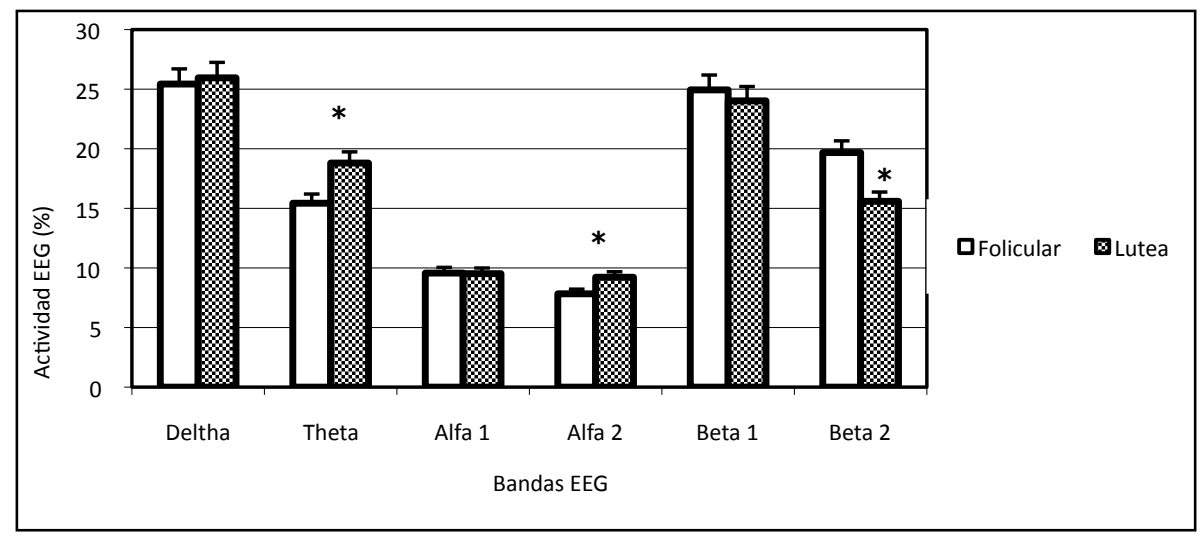

Nota. Las barras muestran la media \pm error estándar del porcentaje de activación de las bandas EEG durante la ejecución de la tarea en las fases folicular y lútea del ciclo menstrual. 
Figura 6. Actividad electroencefalográfica.

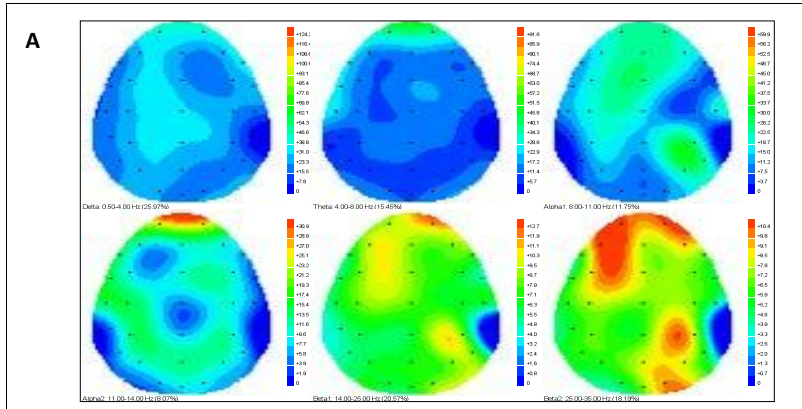

B

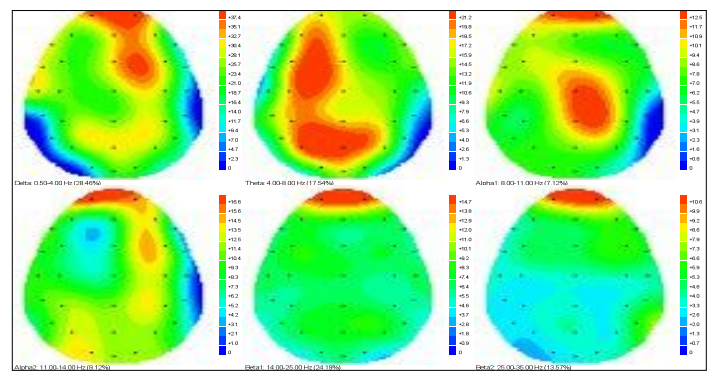

Nota. Las barras muestran las medias de la actividad EEG de las bandas de Delta Theta, Alfa1, Alfa2, Beta1 y Beta 2 de las mujeres con Esclerosis Múltiple en la fase folicular (A) y fase lútea del ciclo menstrual (B).

Figura 7. Actividad electroencefalográfica.

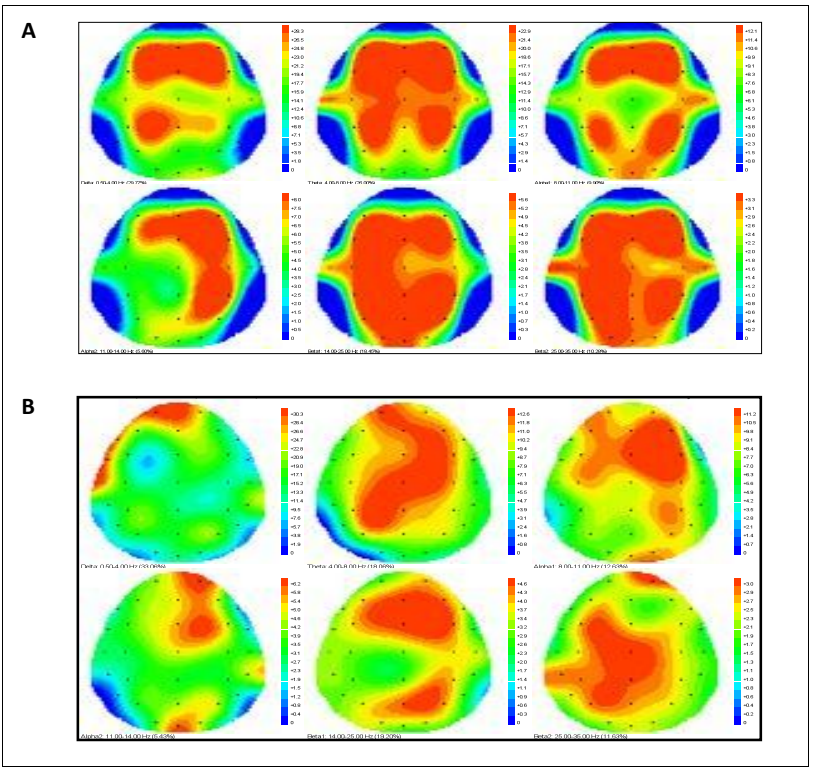

Nota. Las barras muestran las medias de la actividad EEG de las bandas de Delta, Theta, Alfa1, Alfa2, Beta1 y Beta 2 de las mujeres sanas. (A) Fase folicular del ciclo menstrual, (B) Fase lútea del ciclo menstrual.

\section{Discusión}

Nuestros resultados muestran que las pacientes con Esclerosis Múltiple tienen una ejecución deficiente de la tarea de memoria de trabajo, evaluada con la prueba de WCST, durante la fase folicular del ciclo menstrual, cuando los niveles de estrógenos, LH, FSH son altos, y una notable mejoría en la fase lútea, cuando los niveles de progesterona son altos. La deficiencia de la MT observada en estas mujeres, coincide con otros estudios donde evaluaron la MT en pacientes con Escle- rosis Múltiple por medio de diversas pruebas neuropsicológicas, mostrando un procesamiento de información lento en pruebas de memoria de trabajo, sin embargo la ejecución fue similar a la de los controles, lo cual no ocurrió en nuestro estudio (Nocentini et al., 2006, Schulz, et al., 2006) Sin embargo estos autores no distinguieron entre hombres y mujeres; a pesar de que se conoce que las hormonas sexuales influyen en los procesos cognitivos, y que pueden modular la manifestación de los síntomas de la enfermedad (Holmqvist et al., 2006), como se demostrado en mujeres con Esclerosis Múltiple y etapa posparto donde el número de recaídas es más frecuente (Vukusic \& Confavreux, 2006) y las lesiones se hacen más evidentes en imágenes de resonancia magnética asociadas con niveles de estrógenos(Pozzilli et al., 1999), lo cual sugiere que la presencia de estrógenos parece modular los síntomas en estas mujeres (Sandyk, 1996).

Existen pocos estudios de EEG y alteración de la memoria en pacientes con Esclerosis Múltiple, sin embargo se ha demostrado que la actividad EEG de los pacientes con Esclerosis Múltiple y alteración de la memoria, muestra un incremento únicamente de las bandas Delta y Theta en las regiones frontotemporales (Leocani \& Locatelli, 2000), esta ultima relacionada con procesos de memoria de trabajo (Jensen \& Tesche, 2002), lo cual concuerda con nuestros resultados, ya que las mujeres con Esclerosis Múltiple mostraron una mayor actividad de la banda Theta, además de la banda Alfa 2 , mientras que la banda Beta 2 mostró una actividad menor durante la fase lútea, donde la memoria de trabajo fue mejor ejecutada. Lo que nos sugiere un posible efecto compensatorio parcial, tal como refiere Sweet y Rao (2006) (Morgen et al., 2006), ya que la disrupción de las redes neuronales que ocurren como consecuencia del daño en la materia blanca pudieran estar favorecidas por regiones asociadas a la memoria de trabajo, como se confirmo en este estudio, y en la fase lútea hubo una gran activación de la banda Theta; por otro lado se ha reportado que las hormonas esteroideas tienen influencia sustancial en diversas funciones del cerebro, incluyendo funciones cognitivas (Ozahua, 2005), principalmente los estrógenos, ya que se ha descrito que tiene influencia en una gran variedad de estructuras del SNC (Sherwin, 2003), así como sobre el estado de ánimo, la función cognoscitiva y la viabilidad neuronal, incrementado la densidad de las dendritas y evitando el estrés oxidativo, lo que los ha postulado como neuroprotectores (Singh, 2006).

A su vez se refiere que la progesterona tiene implicaciones en la actividad cognitiva (Stein, 2005), sensorial, mecanismos para evitar el daño neuronal (Morali et al., 2005), así como importantes efectos neuroprotectivos (Singh, 2005) ya que induce la síntesis de mielina (Grubber \& Huber, 2003) lo cual facilita la conducción nerviosa soportando que la progesterona juega un rol importante en el cerebro (Shumacher et al., 2004).

\section{Referencias}

Achiron, A., Kishner, I., Dolev, M., Stern, Y., Dulitzky, M., Schiff, E., \& Achiron, R. (2004). Effect of intravenous immunoglobulin treatment on pregnancy and postpartum-related relapses in multiple sclerosis. Journal of Neurology, 251(9), 1133-1137. doi:10.1007/s00415-004-0495-z

Beatty, W. W. (2002). Sex differences in cognitive impairment in multiple sclerosis. The Clinical Neuropsychologist, 16(4), 472-480. doi:10.1076/clin. 16.4.472.13904 
Bruck, W. J. (2005). Multiple sclerosis. Neurology, 252(27), 10-14. doi:10.1212/ 01.wnl.0000176753.45410.8b

Confavreux, C., Hutchinson, M., Hours, M. M. (1998). Rate of pregnancy-related relapse in multiple sclerosis. The New England Journal of Medicine, 339(30), 285-291. doi:10.1097/00019052-200206000-00006.

Drew, P. D. \& Chavis, J. A. (2000). Female sex steroids: effects upon microglial cell activation. Journal of Neuroimmunology, 111(1), 77-85. doi:S01655728(00)00386-6

Duquette, P. \& Girard M. (1993). Hormonal factors in susceptibility to multiple sclerosis. Current Opinion in Neurology and Neurosurgery, 6(2), 195-201. doi:8481563

El-Etr, M., Vukusic, S., Gignoux, L., \& Durand-Dubief, F. (2005). Steroid hormones in multiple sclerosis. Jorunal of Neurological Sciences, 233(1), 49-54. doi: 10.1016/j.jns.2005.03.004

D'Esposito, M., Onishi K., Thompson, H., Robinson, K., Armstrong, C., \& Grossman, M. (1996). Working memory impairments in multiple sclerosis: evidence from a dual-task paradigm. Neuropsychology, 34(9), 853-862. doi: /10.1016/j.bbr.2011.03.031

Everett, J., James, L., Lavoie, E., \& Karyme, K. (2001). Performance of patients with schizophrenia on the WCST. Journal of Psychiatry \& Neuroscience, 26(2), 123-130. doi:PMC1407748

Grubber, C. J. \& Huber, J. C. (2003). Differential effects of progestins on the brain. Maturitas, 46(1), 71-75. doi:10.1016/j.maturitas.2003.09.021

Heaton, R. K. (1981). A manual for the Wisconsin Card Sorting Test. Psychology Assessment Resourses. [Software de cómputo]. Inc. P-O. Box 998, Odessa, Florida.

Holmqvist, P., Wallberg, M., Hammar, M., Landtblom, A. M., \& Brynhildsen, J. (2006). Symptoms of multiple sclerosis in women in relation to sex steroid exposure. Maturitas, 54(2), 149-153. doi:10.1016/j.maturitas.2005.10.003

Jensen, O. \& Tesche, C. D. (2002). Frontal theta activity in human increases with memory load in a working memory task. The European Journal of Neuroscience, 8(15), 1395-1399. doi:10.1046/j.1460-9568.2002.01975.x

Kurtzke, J. F. (1983). Rating Neurologic Impairment in Multiple Sclerosis: an expanded disability status scale (EDSS). Neurology, 33(11), 1444-1452. doi: 6685237

Lang, T. J. (2004). Estrogen as an immunomodulador. Clin Immunol, 113(3), 224230. doi:10.1016/j.clim.2004.05.011

Leocani L. \& Locatelli T. (2000). Electroencephalographic coherence analysis in multiple sclerosis: correlation with clinical neurophyological and MRI findings. Journal of Neurology, Neurosurgery, and Psychiatry, 69(2), 192-198. doi:10.1136/jnnp.69.2.192

Morali, G., Letechipia-Vallejo, G., Lopez-Loeza, E., \& Montes, P. (2005). PostIschemic administration of progesterone in rats exerts neuroprotective effects on the hippocampus. Neuroscience Letters, 382(3), 286-290. doi: 10.1016/j.neulet.2005.03.066

Morgen, K., Sammer, G., Courtney, S. M., Wolters, T., Melchior, H., Blecker, C. R . \& Oschmann, P. (2006). Evidence for a direct association between cortical atrophy and cognitive impairment in relapsing-remitting MS. Neuroimage, 30(3), 891-898. doi:10.1016/j.neuroimage.2005.10.032

Nocentini, U., Pasqualetti, P., Bonacita, S., Buccafusca, M., De Caro, M. F., Farina, D., \& Girlanda, P. (2006). Cognitive dysfunction in patients with relapsingremitting multiple sclerosis. Multiple Sclerosis, 12(1), 77-87. doi:1191/ $135248506 \mathrm{~ms} 1227 \mathrm{oa}$

Ozahua, H. (2005). Steroid hormones, their receptors and neuroendocrine system. Journal of Nippon Medical School, 72(6), 316-325. doi:10.1272/ jnms.72.316

Poser, C. M., Paty, D. W., \& Scheinberg, L. (1983). New diagnostic criteria for multiple sclerosis: guidelines for research protocols. Annals of Neurology, 13(3), 227-231. doi:10.1002/ana.410130302

Pozzilli, C., Falaschi, P., Mainero, C., Martocchia, A., D’Urso, R., Proietti, A., Frontoni, M., Bastianello, S., \& Filippi, M. (1999). MRI in multiple sclerosis during the menstrual cycle: Relationship with sex hormone patterns. Neurology, 53(3), 622-624.

Sandyk, R. (1996). Estrogen's impact on cognitive functions in Multiple Sclerosis. The International Journal of Neuroscience, 86(1-2), 23-31. doi:8828057

Schulz, D., Kopp, B., Kunkel, A., \& Faiss, J. H. (2006). Cognition in the early stage of multiple sclerosis. Jorunal of Neurology, 11, 981-986. doi:10.1007/ s00415-006-0145-8

Sherwin, B. (2003). Estrogen and cognitive functioning in women. Endocrine Reviews, 24, 133-151. doi:10.1210/er.2001-0016

Shumacher, M., Guennoun, R., Robert, F., Carelli, C., Gago, N., \& Ghoumari, A. (2004). Local synthesis and dual actions of progesterone in the nervous system: neuroprotection and myelination. Growth Hormone \& IGF Research, 14(A), 18-33. doi:10.1016/j.ghir.2004.03.007

Singh, M. (2006). Novel mechanisms for estrogen-induced neuroprotection. Experimental Biology and Medicine, 231(5), 514-521. doi:16636299

Singh, M. (2005). Mechanisms of progesterone-induced neuroprotection. Annals of the New York Academy of Sciences, 1052, 145-151. doi:10.1196/annals. 1347.010
Solís-Ortiz, S., Guevara, M. A., Corsi-Cabrera, M. (2004). Performance in a test demanding prefrontal functions is favored by early luteal phase progesterone: an electroencephalographic study. Psychoneuroendocrinology, 29(8), 1047-1057. doi:10.1016/j.psyneuen.2003.10.007

Stein, D. G. (2005). The case for progesterone. Annals of the New York Academy of Sciences, 1052, 152-160. doi:10.1196/annals.1347.011

Sweet, L. H., Rao, S. M., Primeau, M., Mayer, A. R., \& Cohen, R. A. (2006). Functional magnetic resonance imaging of working memory demands among patients with multiple sclerosis. Human Brain Mapping, 27(1), 28-36. doi: 10.1002/hbm.20163

Takao, T., Flint, N., Lee, L., \& Ying X. (2004). 17beta-estradiol protects oligodentrocytes from cytotoxicity induced cell death. Journal of Neurochemistry, 89(3), 660-673. doi:10.1111/j.1471-4159.2004.02370.x

Tomassini, V., Onesti, E., Mainero, C., Giugni, E., Paolillo, A., \& Salvetti, M. (2005). Sex hormones modulate brain damage in multiple sclerosis: MRI evidence. Journal of Neurology, Neurosurgery, and Psychiatry, 76(2), 272-275. doi:10.1136/jnnp.2003.033324

Trenova, A. G., Manova, M. M., Zahariev, Z. I., \& Vasileva, M. (2004). Female sex hormones and multiple sclerosis. Folia Medical, 46(4), 11-15. doi:10.1111/ j.1743-6109.2010.02161.x

Vukusic, S. \& Confavreux, C. (2006). Multiple sclerosis and pregnancy. Revista de Neurología, 162(3), 299-309. doi:RN-03-2006-162-3-0035-3787-101019200601294

Yonker, E., Eriksson, E., Nilsson, L., \& Herlitz, A. (2003). Sex differences in episodic memory: minimal influence of estradiol. Brain and Cognition, 52(2), 231-238. doi:10.1016/S0278-2626(03)00074-5

Zorddrager, A. \& De Keyser, J. (1997). Menstrually related worsening of symptoms in multiple sclerosis. Jorunal of Neurological Sciences, 149(1), 95-97. doi:PII: S0022-510X(97)05396-3 